\title{
Blockchain Technology in Education System
}

\author{
A Survey Examining Potential Uses of Blockchain in Saudi Arabia Education
}

\author{
Afnan H. Alsaadi ${ }^{1}$, Doaa M. Bamasoud ${ }^{2}$ \\ Dept. of IS, College of Computing and Information Technology \\ University of Bisha, Bisha \\ Saudi Arabia
}

\begin{abstract}
The aim of this paper is to review the blockchain technology and its benefits in relations to education system. Blockchain technology is widely researched and highly evaluated and appraised for its unique infrastructure. In general, blockchain researched for its association with Bitcoin and cryptocurrency advantages. In this survey the plan is to conduct a full review of previous literatures focused on blockchain in education systems. Provide overall reviews of blockchain concepts and architecture behind the technology and to examine verification software that used by the technology to improve security and immutability. Brief discussion on the consensus algorithms and hashing function and how these operate and difference type of blockchain will be discussed. In this survey, the existing technology used in Saudi Arabia will be reviewed. Indepth research conducted for over 70 papers, of which 35 noted in this survey. Blockchain emerging promise a real time democracy and justice to all users over the world. Educational Industries said to revolutionize its communication system and accessibility and extend their market globally by widening their admissions and providing secure cost-effective, transparent and immutable communications across their educational platforms.
\end{abstract}

Keywords-Blockchain; certifications; authentication; decentralized-education; transparency; immutability; smart contracts; learning accessibility; fraud prevention; sustainability; ledger; consensus

\section{INTRODUCTION}

Electronic peer- to - peer without any third party is the solution to end double spending [1]. A digital signature is the part of the solution [1]. The authors in [1] invoked hashing by timestamped proof, "the network timestamps transactions by chain them into an ongoing chain of hashing-based proof forming a record cannot be changed”. Additionally, added "the longest chain not only serves as proof of the sequence of events witnessed, but proof that it came from the largest poolof CPU power; keeping long chains to outpace attackers. Blockchain technology first emerged as cryptocurrency technology only, but its popularity increased as the technology addressed other classical problems for the centralized system.

Mainly described as a ledger for digital currency and used for cryptocurrency. This decentralized cryptographic system provided a platform for users to transfers money without necessarily relying on any centralized trusted establishment such as payment services or banks [2].

Blockchain technology becomes popular among researchers and technology developers and cooperation e.g., IBM, Sony and Samsung, immediately after disappearance of its creator [1]. The attention, however, advanced the research of blockchain and brings more critical analysis and examination. Blockchain is not a ledger, it is a journal. Carlson in [3] considered blockchain idea only buried into bitcoin currency and not addressed technology in-depth. Added that "the author has weighed the costs and benefits of transacting with virtual currencies, considered the sustainability of the virtual currency, and contemplated the application of existing regulatory schemes to virtual currency" [3].

This suggesting that blockchain structure is misunderstood and not clearly defined by the authors. However, this is also followed by another Blockchain critics, the authors in [4] suggesting that blockchain technology has a potential to diminish government's authorities by end their role of supervising people's money and subverting critical regulation by cutting out middleman such as large operations and corporations. A full analysis conducted by [5] analyzed the code of law as valuable contribution to blockchain. A critical analysis improves the quality of the research into blockchain technology. On another hand, the strong arguments indicted the usefulness of the technology to be taking seriously by researchers. In [4] the authors added the architectural features of blockchain are the center of the attentions. Blockchain designed in such a way is that it evades the effectiveness of regulation and enforcement [5]. The suggestion made by [4] quoted by [5] mainly focused on the novelty of blockchain as the main point of revolution, rather than using all characteristic structures of blockchain which are not revolution. The points below quoted directly from researcher [5] summarized the basis of blockchain structure suggested to underline both opportunities and threats in the discussion and the reviews:

1) Blockchains are disintermediated and transnational networks, often relying on open-source software protocols.

2) Resilient and tamper-resistant, due to their distributed nature, the consensus mechanisms employed, and the use of hashing.

3) Blockchains are transparent-in the sense that transaction data is authenticated and visible - and the data they contain is non-repudiable (due to the use of publicprivate key cryptography).

4) Characterized by pseudonymity-as they allow transacting parties to participate in the system without disclosing their identity. 
5) Blockchains have incentives and cost structures, e.g., block rewards and mining fees that incentivize and compensate parties maintaining a blockchain-based network.

6) Blockchain are unique, and characteristic is the deployment of consensus mechanisms to coordinate social activity towards an agreement on the situation within the system.

7) Blockchains enable a specific type of "autonomy": they facilitate the execution of software code that is entirely independent of any one party.

(Global Survey for blockchain carried by Deloitte 2020).

Moving forward with blockchain, this technology despite its young age, it has been the center of attention among researchers, analysts, law assimilators and centralized system beneficiaries (middleman). A survey conducted by [6] linked the attention is due to the features presented by blockchain, such as decentralized nature, persistency, anonymity, and auditability properties. To be fully conversant with blockchain concept the followings must be clearly defined and understood [7]:

- Asymmetric key cryptography.

- Consensus algorithms.

- Hashing.

- Consensus.

Blockchain is part of the solution to critical problems of ownership, transparency and can be used in many digital communications as solution to our everyday arising challenges [8].

Existing technology provided education system with numerous advancements of learning environment and productivity, however the authors in [9] cited further area of improvements such smart learning track, where the blockchain technology predicted to play significant role.

A block is an entity (data structure) hold information that present past record and current transactions and keep permanent records of transactions. Each block created through process called mining and each new block connected with its predecessor with cryptographically secured reference shown in Fig. 1. The validation process called the hash function.

How hash function design in blockchain is rather critical process to the success of secured blockchain procedure. For a cryptographic hash function to be considered secure; it must represent a certain characteristics or properties, these features determined the hash function suitability for cryptocurrencies such as Bitcoin and other secured transactions. Thus, the cryptographic hash function is an integral part of the blockchain innovation. It is the main characteristic that gives security capabilities to the processed transactions and making it immutable. Hash function designed to protect data stored in the block [10] and then data in a blockchain distributed across the network of multiple nodes or computers. Thus, blocks data becomes very secure and impossible to be altered retroactively without having to alter the entire chain.

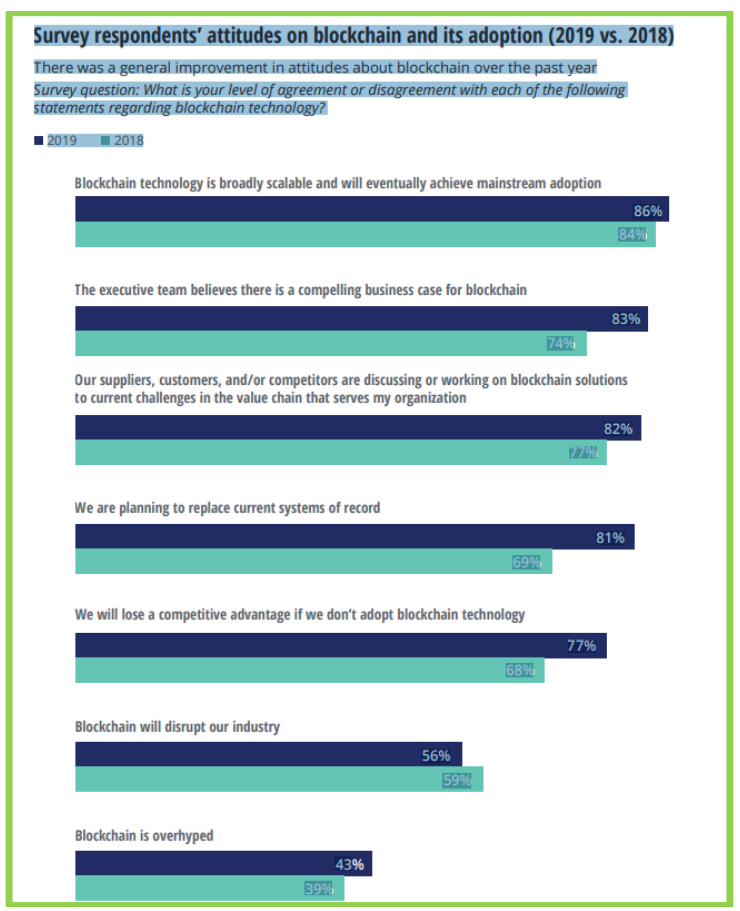

Fig. 1. Blockchain Adoption.

These authentications proofed by mathematical algorithms [11] which deem secure and protected from others. These can be achieved in many ways, but most commonly the use of encryptions and can only be decrypted by legitimate person.

Passing this process, the system integrity can be guaranteed [12] that information has not been modified by unauthorized party. Through this process, system recognizes manipulation and detects it and prevents data corruption. This is how the use of cryptographic hash functions in blockchain works.

There are two significant properties make hash function satisfying [8]:

- Should be fast to compute and strong, and.

- Minimize duplication and data cannot be extracted from its original hash.

The authors stated that: "There are three requirements for practical applications of a hash function for message authentication and digital signatures", the fourth requirement also known as pre-image resistance or one-way property.

\section{A. Blockchain Types}

As shown in Fig. 15, there are three different forms of Blockchain [6].

- Public: Everyone can check the transaction and verify it.

- Consortium: It refers to a node with authority that can be selected in advance, typically has business-tobusiness relationships, the data in blockchain can be open or private, and can be classified as partially decentralized like Hyperledger. 
- Private: This applies to the fact that not every node in the blockchain will participate, with one or more restrictions. The interpretation of firm authority for data management access.

\section{B. How Transaction Complete in Blockchain}

The transaction complete securely between two parties without exchange of identification or third party using public key. Communications are transparent among all networks. Nodes validate transactions and group them in Block [10].

The created block identified by its hash: "a cryptographically unique value calculated on the contents of the block and includes a reference to the hash from the previous block, so that, blocks are linked” [10]. This chain of blocks is thus a record of transactions or a public accounting book (ledger), shared by all the nodes in the network.

Mining is one of the key concepts behind the Bitcoin protocol said [11]. All transaction collected and grouped into block that ready to be linked to its predecessors.

In order to add new block to blockchains, a signature linking the transaction to previous block must be elected [12]. This process involved finding nonce value which is required to satisfy the secure hashing algorithm SHA256. In this surveyor we only briefly explained the functions rather than diving into equations or calculation.

Blockchain technology provides a structural ledger database for storing transactional records known as blocks and links them with numerous databases known as the chain [13]. These immutable records are then signed cryptographically using a distributed consensus or validation protocol [14]. The following processing staging are essential tools to secure blockchain transaction:

1) Education system: technology strength and transformation: Its evidently education system has been evolving and transforming over the past centuries. Institutions and universities provide diverse methods of learning to reach out learners. One of these methods is the distance learning, which dated back since 1873 where first initiated by Professor Ana Eliot Ticknor and then followed by the University of Queensland in Australia in 1911 and the University of South Africa 1946. These humble initiations shaped the distance learning of today. The only difference between now and then was the methods of delivery. Traditionally, learning delivered on campus, certifications received in persons or by post, transcription and copy of credential kept with registrars, but this paradigm have been shifting gradually to online educations and communications: All communications currently handled online. From entry applications, payment system, and learning, handling coursework, and submitting assignment and communicating with tutors all completed through universities platforms.

The paradigm shifting in educations often appraised for its efficiency, increased productivity and improved learning outcomes. A research conducted by Bill\& Melinda Foundation on positive distance learning and how technology advanced learned outcome.

The report conducted by [15] expressed positive outcome for learner at the time, it was a revolution concept to create such "learning environment that student in rural areas had expanded learning opportunities in a variety of subject.” [15]. The Impact of digital technology is countless and how this technology improves learning outcomes is incalculable.

A research conducted in relation to digitalization. "In terms of education this means we should create Knowledge that is accessible virtually with the focus on the student”. This means students are build and developed from all areas and these facilities aid their learning.

Rather those days where students she/he travel distances or encountering lock of access to documents or physical visit to library and time restrictions to information.

The authors in [16] added virtual access is achieved through Internet or Intranets, facilities such as "e-mail, web notices, discussion forums and video conferencing allow a student to access information without visiting the physical location of delivery”. A typical interactive e-learning system will have these characteristics and thus demonstrates the paradigm shift” [16].

2) Education system: technology weakness and volatility: Despite the enormous benefits of technology in education and the positive contribution achieved, technology comes with some downside risks and must be appropriately evaluate [17]. In relation to how technology apprised, and debates the author, highlighted that the impact of technology in education should appropriately addressed both strength and weakness. Unforeseen its potential risks will hinder the quality of the any review [17].

A recent public awareness publishing; highlighted these facts: Data published by [18] highlighted critical information into how criminal may damage universities infrastructurebased knowledge and steal significant data that can be harmful to countries' economy.

“In 2018 researcher discovered logging pages for 300 fake websites and login pages for 76 universities across 14 countries". "And between 2013 and 2017, accounts of more than 100,000 professors worldwide stolen, and led to the loss of more than 30 terabytes of academic data and intellectual property”. [18]. As we moving to cloud space and more online present via working groups or communicating online, there is urgency to seek more security and proof systems to convey information and communication through.

3) Education: system venerability (centralized cloud space): Cyberspace attacks can be permanently damaging to system and can be irreversible. The authors in [18] Highlighted reasons behind such attacks that could be and not limited to the followings:

- For financial gain, criminals can sale critical data and research information and breakthrough to hostile 
enemies or competitors to publish dedicated researcher findings and claim ownerships.

- Steal critical information on national infrastructures research for knowledge advancement, substage strategic planning and future projects.

- Malicious attackers can damage important files and documents and can navigate through to gain full access to intellectual properties.

- Talents at universities can be targeted and their activities monitories by infiltrator.

- Undermine national security and economic prosperity.

4) Education: blockchain the transformation promise: Blockchain Technology its innovative disruptive system with prominent potentials, fully authenticated and transparent, economically viable and with direct and indirect benefits to support sustainable educational system for all.

Recent survey carried by [19] on usability and impact of blockchain adoption including positive impact and barriers of use. Lack of knowledge of the technology and comparison with the existing system showed impactful results. The following Fig. 1 to 6 shows Survey results:

(Global Survey for blockchain Deloitte 2020).

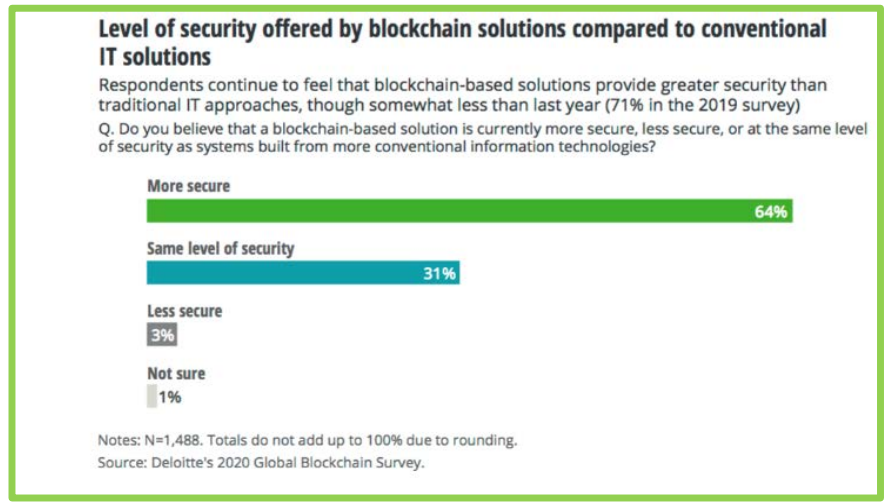

Fig. 2. The Level of Security vs IT Solution.

Views of blockchain's relevance within organizations
Most respondents consider blockchain important or even critical to their top priorities
Survey question: Which of the following best describes how you currently view the relevance of blockchain to your
organizotion or project in the coming two years?
Critical, in top 5 strategic priorities
Important but not in top 5 strategic priorities
Relevant but not a strategic priority
Not relevant
$3 \%$
Unsure/no conclusion
$3 \%$

Fig. 3. Blockchain Significant Advantages over Existing System.

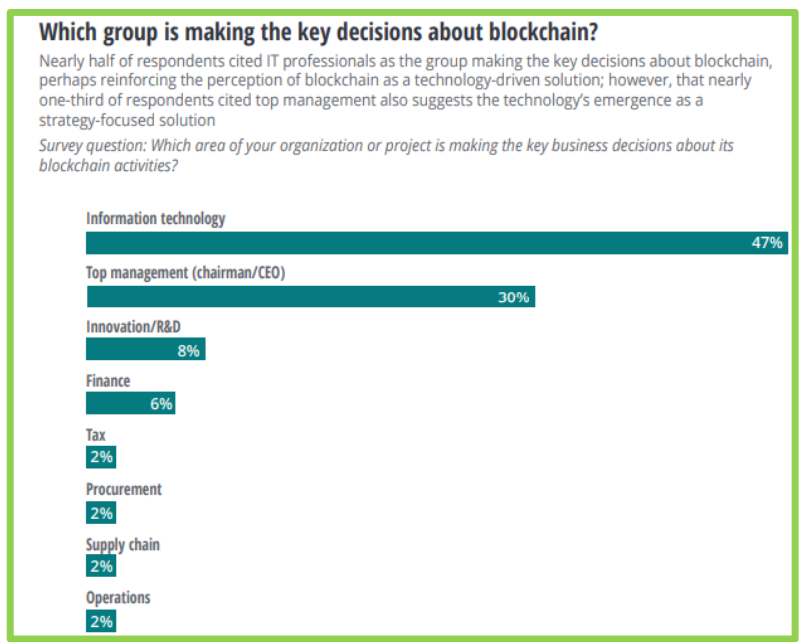

Fig. 4. Global Survey for Blockchain Carried by Deloitte 2020.

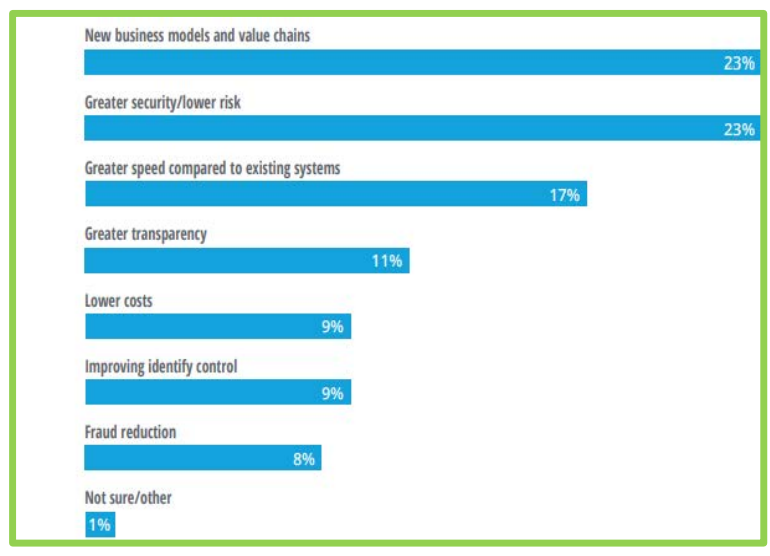

Fig. 5. Decision Maker on Organization.

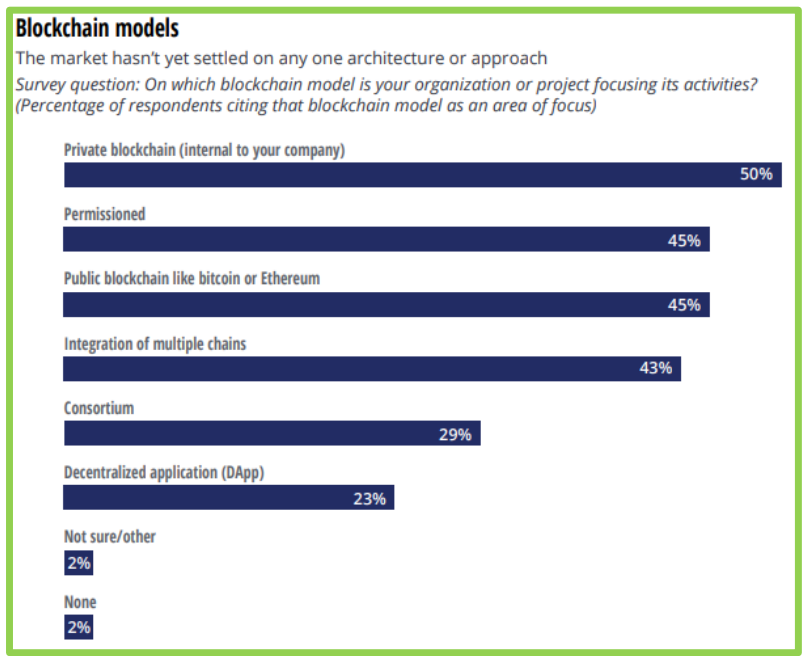

Fig. 6. FBlockchain Model.

5) Education: blockchain technology: The first case reviewed is a Cambridge, Massachusetts education model: "MIT has formed the Digital Credentials Consortium an international network of universities to develop a shared system for digital academic credentials [20]. Public ledger 
technology enables us to develop new tools, standards, and strategies to store and manage digital academic credentials. Certificates can be cryptographically signed and tamper-proof. They can represent or recognize many different types of achievements [21]. DCC was founded in 2018 by leading universities with expertise in the design and verification system.

Academia of DCC raised questions about the overall system of trusted verification and authentication of learning and credentials; and there was a clear concern about the way credential issuing and transcription managed, after consultation, consortium strongly agreed that there is an urgency to renew system. Benefits for learners:

- Maintain a compelling verifiable record of student lifelong learning achievements to share with employers in a trusted manner.

- A safe and secure digitally transferred credential with no extra fees.

Available secure stored data from multiple educational institutions and credential. A team from learning machine and MIT media lab creates "blockers" an open standard platform to issuing and verifying blockchain certificates. DCC highlighted future project planning [21], "we are exploring how recent advances in public key infrastructures, public ledgers, and blockchains can be used to rethink the way we recognize and transact with academic achievements”.

Digital Credential Consortium is a powerful organization with unique strength of intellectual talents. "The infrastructure for design and governance of academic credentials has been set a sight and credentials now transformed into tokens of social and human capital that can create new opportunities for participation in educational industry" [21].

"Real-life use case: More than 600 of 2018 MIT graduates chose to receive a digital version of their diplomas on Blockcerts' blockchain. Consequently, the students' academic records will be stored forever, and future employers can immediately verify them" [20].

Organization and world's fined universities and institutions are benefiting from blockchain technology and building searcher on how to completely adopt this technology. In this survey, several universities will be reviewed:

a) Curriculum: Smart curriculum design is one of the most researched subjects these days. Mainly associated with blockchain technology and the usefulness of its features. In [22], the QualiChain platform that will offer blockchainenabled verification of education and other credentials as well as data analytics and decision support for process optimization [22].

Permanence of blockchain record, smart contracts these features seen as attractive tool for education commissioners [23]. These features make blockchain technology-based products or services significantly different from previous internet-based commercial developments [23]. The authors in [23] addressed the lack of knowledge about the social advantages and potential for blockchain in education by stakeholders. The authors highlighted the benefits blockchain in comparison with existing system: shown in Fig. 7.

- Self-sovereignty, i.e. for users to identify themselves while at the same time maintaining control over the storage and management of their personal data.

- Trust, i.e. for a technical infrastructure that gives people enough confidence in its operations to carry through with transactions such as payments or the issue of certificates.

- Transparency \& Provenance, i.e., for users to conduct transactions in knowledge that each party has the capacity to enter that transaction.

- Immutability, i.e. for records to be written and stored permanently, without the possibility of modification.

- Disintermediation, i.e. the removal of the need for a central controlling authority to manage transactions or keep records.

- Collaboration, i.e. the ability of parties to transact directly with each other without the need for mediating third parties.

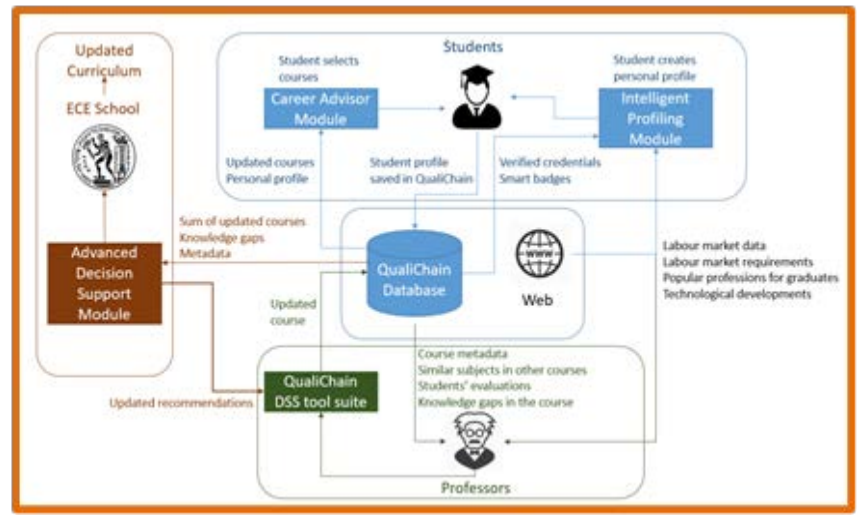

Fig. 7. Education Curriculum.

b) Certification: The Hyperledger Fabric is an open source blockchain platform (refer to Fig. 8 and 9).

Hyperledger Fabric Architecture and permits for faster blockchain network. The application connects with blackchin via REST web, APIs and by using HTTP and JSON data format [25].

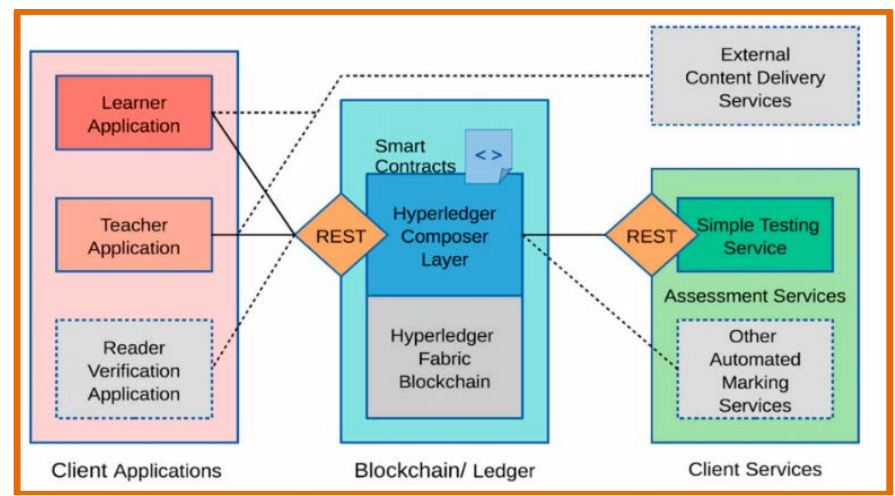

Fig. 8. Hyperledger Structure. 


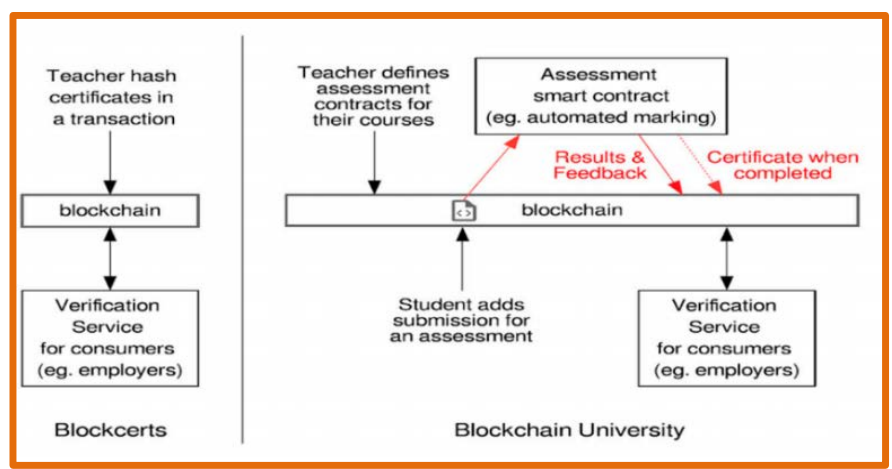

Fig. 9. Blockcert (Blockchain Certificates) for University.

c) Smart Contract: Smart contract covers ranges of facilities in crypto-educations system, from enrolling into course to coursework evidenced based, timeline tracking, helps both tutors and learners' opportunities to manage datelines, fast enquiry and verifications, track of students' learning progress etc.

Transaction processed in transparent and scalable manner to insure full transparent [24].

- Summarized five Timestamped logs.

- Cryptographic \&Timestamped Logs.

- Cryptographic hash function.

- Timestamped append-only Logs.

- Block headers \& Merkle Trees.

d) Immutability: Technology profoundly changed educational system but "the way we issue and managed academic credentials, which represent learning outcomes and achievements, has not yet taken advantage of the possibilities of digital technology". The blockchain can resolve these issues and provides data access to universities in permissionsless or permissioned (private permissioned -loop) such as peers control access described by [25]. Peers control access model discussed and adopted worldwide by large organization and private consortiums. Is less complex to use and tailored to specific application.

"The adoption of cloud computing is in line with the objective of saving resources/saving costs and improving the interaction between students and the educators. "The future of How Blockchain deliver its activities in absent of administrators and how tasks, sequences and process occurred in a simple structured and intelligent way and protect itself without external forces.

Although blockchain structured as database or system platforms blockchain is also machine learning, deep learning and autonomous systems simply does everything and can do everything. Large corporation making the utmost benefits of blockchain, Sony, IBM, IEEE, etc.

e) Cybersecurity: Security is one of essential unique feature of blockchain, hence each step carried with security in mind. These three-encryption control mechanisms are indispensable for blocks encryptions:
- The system randomly generates a 32-bit whole number known as (nonce) this number only use once, which is then added to a hashed to encrypt. These steps are essential to protect data from cypher attached.

- A block header hash is then generated.

- Hash is a 256-bit cipher merged with the nonce. Data kept within the block.

The hash function key is one of the most important elements in blockchain structure and Merkle tree. These sets of structure's differentiate blockchain uniqueness [14], shown in Fig. 14.

f) Sustainability: Sustainability is the future business. The subject of digitalization and blockchain adoption is not completed without in depth instigation into sustainability, and business continued and its efficiency and consumption. Given the governing authorities and strategies taken by a given government, compliance is needed to carefully address incompatible innovation and instigate threats to digital sustainability and assessment, in particular the role of politics. [26]. Shown in Fig. 10, adaptive governance can be defined as the adjustment of regulatory rules and practices to incorporate new data and to balance the risks and benefits of a given activity.

g) Consensus: It is important part of validation by all participants in this mechanism Blockchain enhances trust across a business network". With blockchain trust comes natural within the network community because it provides cryptographic proof over a set of transactions.

“Because the transaction can't be tampered with and are signed by the relevant counterparties, any corruption is readily apparent”. Blockchain is sustainable and its existence will be continued since it's an independent and not own by any vendor.

Organizations like the IEEE developing courses in many areas of education to sell to corporate and other societies. Blockchain strengthen existing standards. "As a recordkeeping strategy, blockchains pose a novel way to track whether participants are complying with standards that they publicly claim". [4]. IEEE taking full advantageous of blockchain smart processing tools and tight security.

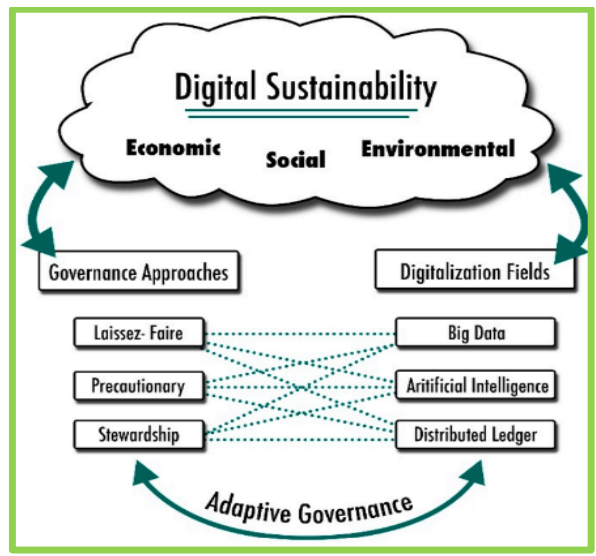

Fig. 10. Digital Sustainability. 
h) Consensus process: Blockchain and our smart credential platform in the education sector will explore areas of automatic recognition and attribution or transfer of credits, verifying accreditors lifelong records of learning, student grants and funding also can leverage sovereign identities for verifying students and even educators" [27]. Sony creating trusted platform using blockchain. "We will be demonstrating how blockchain will become the future of maintaining and managing transcripts and high security data in education" [ 27].

IBM [14] and Linux Foundation also in partnership with Sony developed 'Hyperledger'. These initiatives enables multiple institutions to add transcription and student's achievement on a ledger records for a lifetime. "Blockchain technology makes it possible to associate these types of data with individuals" [20]. The authors added "This will enable people to handle their own academic progress record, and to have control over how to improve their learning cycle, for example. I believe this will change education as we know it and make it substantially more efficient,” [20]. As a global leader in this field there is no doubt his input will shape the world of digital education. IBM hyper ledger offer both public and private tailored access to its end-users.

Blockchain technology spreading fast and among large and reputable universities and institutions, this not to underestimates the challenges emerging from researchers challenging blockchain, efficient and scalability of the system, [28]. In a permission-based system, decision taking to for an example, how to ignore malicious nodes and mining processing executions computation. There also concern about designing of smart contract based blockchain application.

Many enterprises integrate blockchain with their systems for the benefits of the blockchain. Despite its strength, blockchain has some challenges in security, privacy, scalability, and other issues [28]. Although blockchain breakthrough solves major issues, certain areas within blockchain deserve further attention and scrutiny. There should be more critical analysis and evaluations, to ensure earl rectification of upcoming problems. Blockchain is a transformation that changed the world for better, despite the slow progress of these technologies beside educational system.

Other technologies emerging from blockchain can bring some social justices to humanity. The past decades technology is controlled by only half of the world and the rest are poor, deprived and disfranchised. Half of the world still does not connect to basic elements of technology for example electricity, education and healthcare, the main reason for these is those intermediaries' systems of greed.

Promotes transparency, "You build peer-to-peer selfsustaining applications that can stand its ground. From destabilized governments to supply chain vendors, use cases of blockchain are endless. Control over personal information is vital and can be successfully addressed using blockchain" [29]. Blockchain story is not going away, and its strength and capability is growing fast, "A billion dollars in venture capital has flowed to more than 120 blockchain-related start-ups, thirty of the world's largest banks have joined a consortium to design and build blockchain solutions, Nasdaq is piloting a blockchain-powered private market exchange and Microsoft has launched cloud-based blockchain-as-a-service" [30].

6) Digital education system: Saudi Government boosted its digital Education system and made enormous transformation by implementing a set of EdTech infrastructure 2017. Future Gate is a country-wide, large-scale initiative of Tatweer education and part of KSA vision for 2030 developments is to focus on digital literacy skills of 21st century.

7) Current education system in Saudi Arabia: Saudi Arabia student's outbound policy decreases and now reverses the equation by investing in their educational system and opening new opportunities of international student studying in the Saudi Arabia. Recent reports by WENR [32] showed slow on Saudi's outbounds students decreases and inbound increases both by national and international students' seeking education in Saudi Arabia. "The expansion of Saudi Arabia's higher education system and the construction of more universities in recent years has helped accommodate a rising inflow of students from other countries, resulting in a high inbound student mobility ratio of 4.6 percent between 2008 and 2017" [33].

Since 2019 Saudi Arabia open new venues of shared researchers and collaborations with international foreign universities to stimulate students' exchange. This is seen as positive steps forward to Saudi openness and commitment to educations. Fig. 11 and 12 show statistic carried by WENR.

Now Saudi Arabia authorized foreign universities to set up Campuses and there is strategy to slow outbound students' mobility. This certainly reduces outbound costs and strengthens inbound prosperity. This is also an opportunity for Saudi to adopt blockchain technology for its international exchange and by slowing outbounds students and bring in international universities can provide cost effectives digital education at home and make double win saving and attracting inbounds students by using Blockchain Technology to facilitate and improve education system [32].

\section{International Schools in Saudi Arabia by Regional Comparison (2017)}

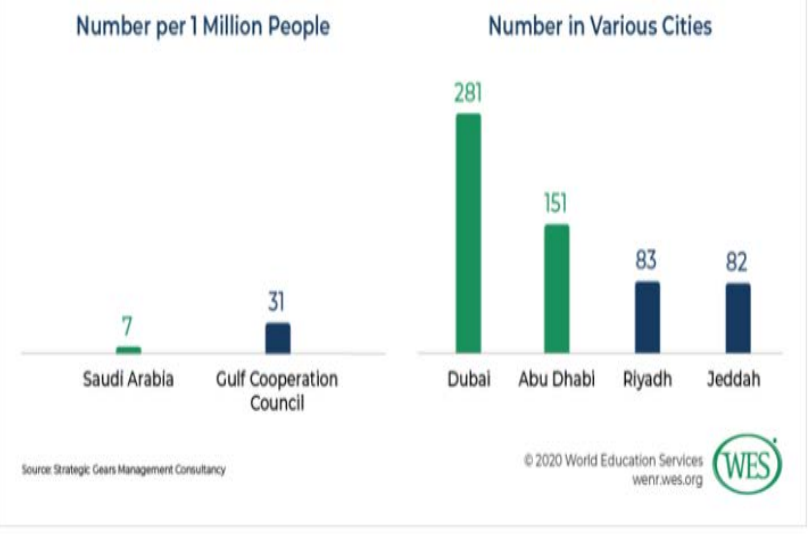

Fig. 11. Statistics of Outbound Saudi Arabian Students. 


\section{WES $17=$ World Education News + Reviews}

\section{EDUCATION IN SAUDI ARABIA}

\section{QUICK FACTS}

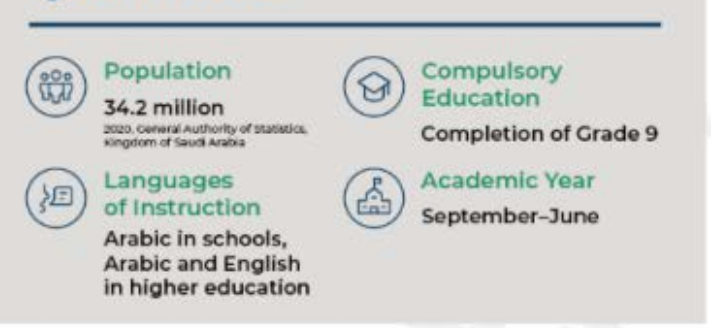

\section{SAUDI ARABIAN INTERNATIONAL STUDENTS} IN THE U.S.
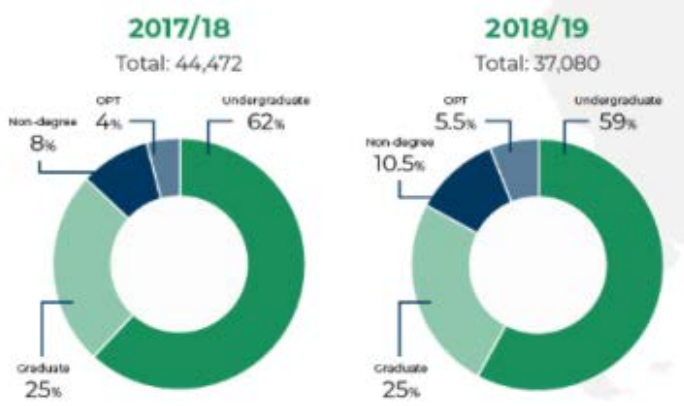

Source institute of international Educat:

Fig. 12. Saudi Arabian International Students in The U.S.

Blockchain Potential Benefits and Impact on Saudi Arabia Education, as shown in Fig. 13:

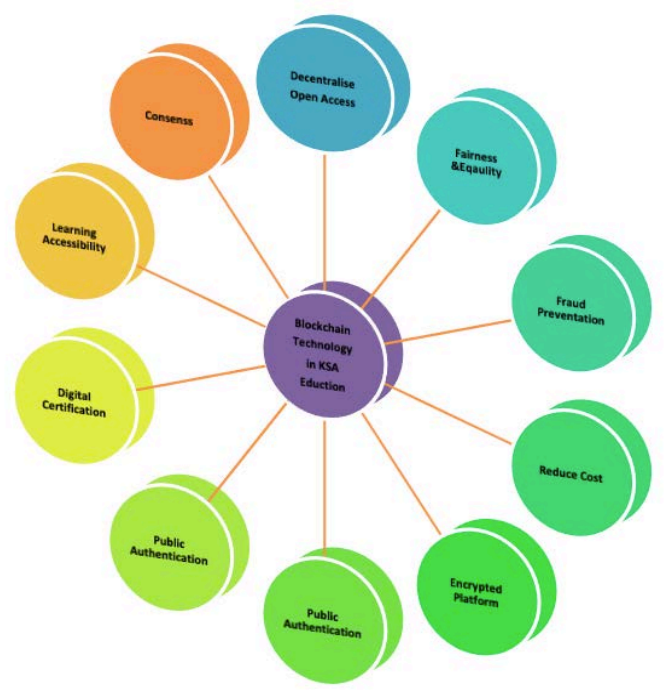

Fig. 13. Proposal Blockchain Potential Benefits to Saudi Education.

\section{SWOT ANALYSIS OF USING BLOCKCHAIN TECHNOLOGY}

\section{A. Strength of using Blockchain Technology}

1) Blockchain is decentralized data setting is incorruptible and consistently reliable online database registry of various digital transactions where participants can modify the data by following a process of approval procedures: this called consensus and, in this process, all nodes in the network or majority must agree to transactions.

2) Blockchain is an encrypted system that uses different styles of encryption and hash to store data in protected databases. The system distributes these data records over various nodes and forms a consensus on the position of the data they contain that makes difficult to corrupt.

3) Blockchain data and information not stored in local system or cloud space but in multiple nodes tight secured. Main principle of decentralization, information not kept in one space and data is transparent [21].

4) Smart contracts are computer protocol that facilitated and enforce negotiation. Can be partially or fully and selfexecuted and self-enforced. In Ethereum these contracts have the ability to communicate within their internal storage and analyses, evaluate and send message to trigger execution. Smart contracts offer valuable advantages: such as enforcement, management, and payment, and performance, these aspects must be satisfied in fast and effective manners without third party authorization [21].

\section{B. Weaknesses associated with the use of Blockchain Technology}

1) Existing centralized vendor managed system both traditional and cloud-based structures offer services at sum costs and provides in return, computing services that includes software, storage, servers, database, networking, analytics reports, intelligence reports and flexible utilization of resources [18]. Thus, makes competition to newly emerged blockchain harder or less favorable to businesses comfortable with these facilities.

2) Blockchain has some challenges in security, privacy, scalability [33].

3) Blockchain is here and to stay and to influence major actors globally, it has been recognized and gradual implementation across industry spreading fast [35] (refer to Fig. 16 blockchain mind map).

4) Old system offers history of services and builds trusts among users and vendors common interests. Offer transformation and rapid innovation to its end-users.

5) Traditional and newly formed cloud space, structure of database stored locally and on cloud storage with simple procedures access. In centralized system (data stored in a company's data centers). Offer tailored and optional transparency and stored data can be visible to public or private as requested. 


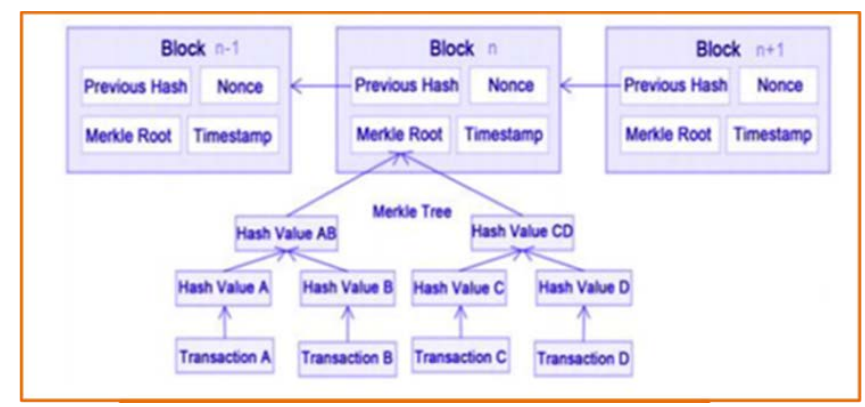

Fig. 14. Hash Function.

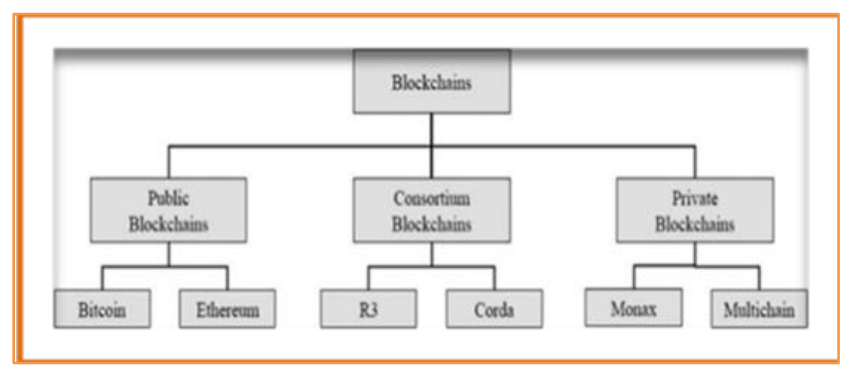

Fig. 15. Types of Blockchain.

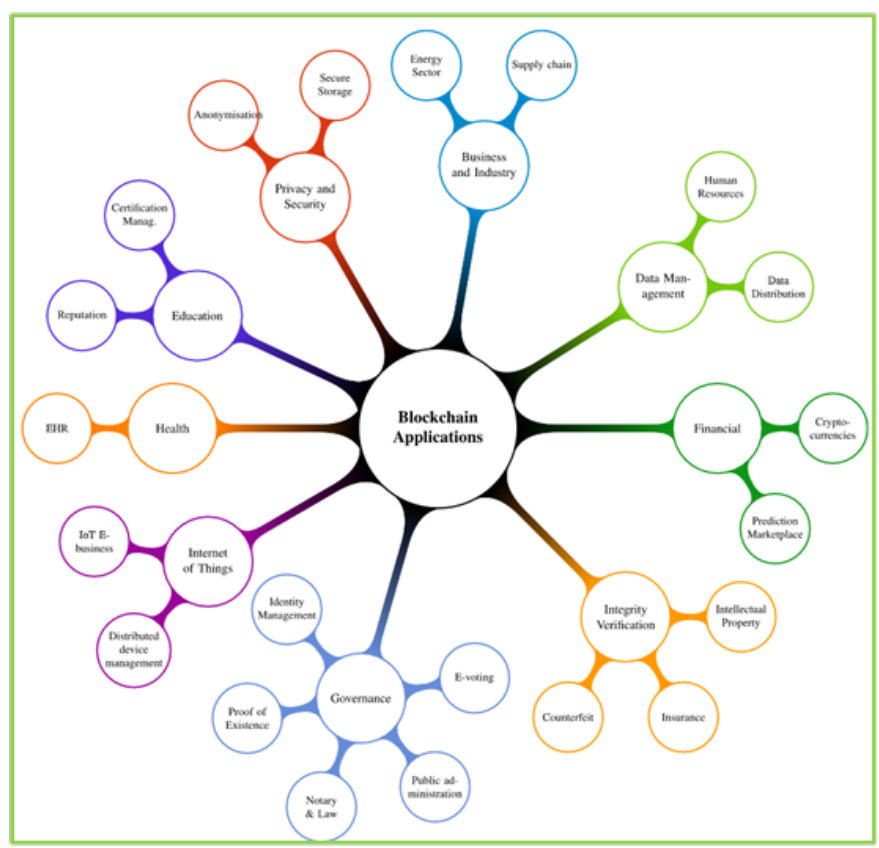

Fig. 16. Mind Map Abstraction of different Types of Blockchain Applications.

\section{Opportunity Associated with Blockchain Technology}

1) Recoded ledger and immutability.

2) Tight cryptography (using hash function).

3) Decentralizes and open access.

4) Availability.

5) Strength distributed resilience and control.

6) Decentralized network and open source.

7) Asset's provenance.

8) Dynamic environment.
9) By removing intermediaries and delivering a dependable shared view of permissioned data, blockchain could:

10)Reduce costs effectively: (e.g. Administrations, university running costs, Third party and students).

11)Speed up settlement (e.g. faster validation, digital enrolment verifications, and certification).

12)Increase resilience (e.g. no single point of failure).

13)Improve transparency (e.g. easier to monitor).

\section{Threat}

Threats and risks by [31] analysts seem glomming but effective as well. Ledger conflicts competition, Lack of ledger interoperability Lack of inter-ledger governance and limitation of smart contract coding programming.

\section{CONCLUSION}

To conclude this study, blockchain has reached its maturity in education system globally, despite the speed at which educational establishments are moving towards complete change of system and full blockchain technology adaptation. The fact Blockchain still available for adaptations, those who start first will benefit more. The scale of emerging educational platform using blockchain Technology growing fast and making use of all benefits ranging from smart contracts system of payments to Id identification and token and students learning authentications, thus the story of blockchain is growing and staying.

World Class education Institutes are using the technology to their advantageous, for instance the powerful educational Institutes such as Cambridge, MIT, are teaming up to create Blockcerts, an open standard platform for creating and issuing verifying certificate's. In 2018 MIT 600 graduates accepted digital version of their diplomas on Blockcerts blockchain, other universities and educational. A consortium consisted of top world university called (DCC) Digital Credential Consortium issuing both credential and transcripts already in place. During this study research encounter several papers discussing the weakness of this technology and focus was on the scalability issues and shortage of skills rather than major structural issue with blockchain frameworks. These voices raised concerns in relation to scalability and proof-work, others focus on privacy and closed loop system. Blockchain has fruitful future and benefits to all industry including major benefits to education system. This study has its limitation; however a better review in future will cover most areas in more prices and systemic approach. Blockchain offers real promise to all industries without exceptional. This surveyor focused on blockchain functionality and potential for its education system. Most research focused on the main issues blockchain differentiates itself from the reset of the other technology, which is the decentralization and immutability and transparency and more advancement into certification platform such as Hyperledger's. There should be further indepth research mainly focused in how these platform developed and functionality i.e. prototyping, product testing and survey on privacy vs openness of decentralization. Majority of research developed built on previous literatures and limited further opinions or new development which seems 
closed loops as if all researchers working from one point of view. There is no underestimation of the prefund efforts made by the blockchain community of research. New development need and research guidance and direction also missing.

\section{REFERENCES}

[1] Nakamoto, S., \& Bitcoin, A. (2008). A peer-to-peer electronic cash system. Bitcoin.-URL: https://bitcoin. org/bitcoin. pdf, 4.

[2] Sabry, S. S., Kaittan, N. M., \& Majeed, I. (2019). The road to the blockchain technology: Concept and types. Periodicals of Engineering and Natural Sciences (PEN), 7(4), 1821-1832.

[3] Kristofer J Carlson, October 2018, https://www.researchgate.net/ publication/328581315_The_Nakamoto_Blockchain.

[4] De Filippi, P. D. F. (2018). Blockchain and the law: The rule of code. Harvard University Press.

[5] Quintais, J., Bodó, B., Giannopoulou, A., \& Ferrari, V. (2019). Blockchain and the law: A critical evaluation. Pedro Quintais, B. Bodó, A. Giannopoulou, \& A. Ferrari (2019). Blockchain and the Law: A Critical Evaluation. Stanford Journal of Blockchain Law \& Policy (2), 1.

[6] Mohanta, B. K., Jena, D., Panda, S. S., \& Sobhanayak, S. (2019). Blockchain technology: A survey on applications and security privacy challenges. Internet of Things, 8, 100107.

[7] Sabry, S. S., Kaittan, N. M., \& Majeed, I. (2019). The road to the blockchain technology: Concept and types. Periodicals of Engineering and Natural Sciences (PEN), 7(4), 1821-1832.

[8] Sharmila, K., Kamalakkannan, S., Devi, M. R., \& Shanthi, M. C. (2019). A comprehensive study on blockchain with its components, taxonomy and consensus.

[9] Hashmani, M. A., Junejo, A. Z., Alabdulatif, A. A., \& Adil, S. H. (2020, October). Blockchain in Education-Track ability and Traceability. In 2020 International Conference on Computational Intelligence (ICCI) (pp. 40-44). IEEE.

[10] Pina, A. R. B., Torlà, C. B., Quintero, L. C., \& Segura, J. A. (2017). Blockchain en Educación: introducción y crítica al estado de la cuestión. Edutec. Revista Electrónica de Tecnología Educativa, (61), a363-a363.

[11] O'Dwyer, K. J., \& Malone, D. (2014). Bitcoin mining and its energy footprint.

[12] Sobti, R., \& Geetha, G. (2012). Cryptographic hash functions: a review. International Journal of Computer Science Issues (IJCSI), 9(2), 461.

[13] Morgen Peck, Freelance Technology Writer, A White Paper on "Reinforcing the links of Blockchain " in IEEE Spectrum Magazine special edition "Blockchain World”, November 2017.

[14] Doaa Mohey El-Din M. Hussein, Mohamed Hamed N. Taha, Nour Eldeen M. Khalifa Faculty of Computers and Information Cairo University Egypt, (IJACSA) International Journal of Advanced Computer Science and Applications, Vol. 9, No. 8, 2018

[15] Jeffrey T. Fouts, 2000, Bill and Milenda Gate Foundation.

[16] Wikramanayake, G. N. (2005). Impact of digital technology on education.
[17] Alhumaid, K. (2019). Four Ways Technology Has Negatively Changed Education. Journal of Educational and Social Research, 9(4), 10-10.

[18] The cyber threat to Universities. (2021). Retrieved 2 April 2021, from https://www.ncsc.gov.uk/report/the-cyber-threat-to-universities.

[19] Deloitte blockchain Global Surevy, 2019-2020. from https://www2.deloitte.com/content/dam/Deloitte/se/Documents/ris k/DI_2019-global-blockchain-survey.pdf.

[20] Masaaki IsozuP resident, Sony Global Education, Inc. https://www.sony.com/en/SonyInfo/sony_ai/blockchain.html.

[21] Nadeem bhati, Hyperledger, 20 May 2019, https://www.hyperledger.org/blog/2019/05/20/developer-showcaseseries-nadeem-bhati-high-school-technology-services.

[22] Kontzinos, C., Markaki, O., Kokkinakos, P., Karakolis, V., Skalidakis, S., \& Psarras, J. (2019). University process optimisation through smart curriculum design and blockchain-based student accreditation. In Proceedings of 18th International Conference on WWW/Internet.

[23] Grech, A., \& Camilleri, A. F. (2017). Blockchain in education. Luxembourg: Publications Office of the European Union.

[24] Herian, R. (2018, December). Legal recognition of Blockchain registries and Smart contracts. EU Blockchain Observatory and Forum.

[25] Ullah, N., Mugahed Al-Rahmi, W., Alzahrani, A. I., Alfarraj, O., \& Alblehai, F. M. (2021). Blockchain Technology Adoption in Smart Learning Environments. Sustainability, 13(4), 1801.

[26] Van Oudheusden, M. (2014). Where are the politics in responsible innovation? European governance, technology assessments, and beyond. Journal of Responsible Innovation, 1(1), 67-86.

[27] Sony Global Education, 2017, Creating a Trusted Experience with Blockchain, https://blockchain.sonyged.com

[28] Gupta, M. (2020). Blockchain for Dummies (3rd ed.). Hoboken: John Wiley \& Sons, Inc.

[29] Alajmi, Q, Sadiq, A. Kamaludin, A., \& Al-Sharafi, M. A. (2017, May). E-learning models: The effectiveness of the cloud-based E-learning model over the traditional E-learning model. In 2017 8th International Conference on Information Technology (ICIT) (pp. 12-16). IEEE.

[30] Building the digital credential infrastructure for the future. (2021). Retrieved 1 April 2021, from http://philippschmidt.org/articles/2020-01White-paper-building.

[31] David Furlonger, Ray Valdes, Published: 03 March 2017, https://blockcointoday.com/wp-content/uploads/2018/04/PracticalBlockchain_-A-Gartner-Trend-Insight-Report.pdf.

[32] Sidiqa Allahmorad, and Sahel Zreik, World Education Services April 9, 2020,

[33] Sanka, A. I., Irfan, M., Huang, I., \& Cheung, R. C. (2021). A survey of breakthrough in blockchain technology: Adoptions, applications, challenges and future research. Computer Communications.

[34] Schatsky, D., \& Muraskin, C. (2015). Beyond bitcoin. Blockchain is Coming to Disrupt Your Industry.

[35] Casino, F., Dasaklis, T. K., \& Patsakis, C. (2019). A systematic literature review of blockchain-based applications: current status, classification and open issues. Telematics and informatics, 36, 55-81. 